\title{
PRODUCTIVIDAD DE UN HARVESTER EN RALEOS DE PLANTACIONES DE PINOS EN EL NORESTE ARGENTINO
}

\section{HARVESTER PRODUCTIVITY IN THINNING PINE PLANTATIONS IN NORTHEASTERN AR- GENTINA}

\author{
Eduardo Hildt ${ }^{1}$ Patricio Miguel Mac Donagh ${ }^{2}$ Francisco Ferrando Somma ${ }^{3}$ Diego Alegranza ${ }^{4}$ Daniel \\ Durán ${ }^{5}$
}

\section{RESUMEN}

Se analizó la productividad de segundos y terceros raleos en plantaciones de Pinus taeda y Pinus elliottii var. elliottii x Pinus caribaea var. hondurensis realizados con harvesters de ruedas en el Noreste Argentino. Los árboles cosechados en los segundos raleos presentaron un volumen medio de $0,43 \mathrm{~m}^{3}$ para Pinus taeda de 12 años y $0,36 \mathrm{~m}^{3}$ para pino hibrido de 10 años, mientras que en los terceros raleos este valor fue de 0,71 $\mathrm{m}^{3}$ para Pinus taeda de 13 años y $0,75 \mathrm{~m}^{3}$ para pino hibrido de 15 años respectivamente. Se desarrollaron modelos de tiempos parciales para cada una de las actividades que componen el ciclo operacional y se determinó la influencia del tipo de raleo, especie cosechada, número de productos elaborados, volumen de los árboles y forma de volteo, sobre el tiempo insumido por cada actividad. Los modelos ajustados fueron integrados en un modelo de productividad efectiva consiguiéndose un coeficiente de determinación ajustado $\mathrm{R}^{2} \mathrm{Aj}$ de 0,61. El volumen de los árboles resultó significativo para explicar la duración de todas las actividades evaluadas, siendo especialmente determinante en el volteo de los árboles, su desrame y trozado. El tipo de raleo afectó al tiempo de ubicación, mientras que la especie afectó al tiempo de desrame y trozado. La elaboración de hasta tres productos diferentes requirió un mayor tiempo destinado al desrame y trozado. Ocurrió un aumento de la cantidad de productos cuando se realizaron terceros raleos sobre pino hibrido, respecto Pinus taeda.

Palabras clave: Harvester de ruedas; Pinus taeda; Pino híbrido.

\begin{abstract}
The productivity of second and third thinning operations in Pinus taeda and Pinus elliottii var. elliottii $x$ Pinus caribaea var. hondurensis performed by wheeled harvesters in northeastern Argentina was analyzed. Trees harvested in the second thinning had an average volume of $0.43 \mathrm{~m}^{3}$ for a 12 years old Pinus taeda stand and $0.36 \mathrm{~m}^{3}$ for a 10 years hybrid pine stand, while in the third thinning the tree volume was $0.71 \mathrm{~m}^{3}$ for a 13 years old Pinus taeda and $0.75 \mathrm{~m}^{3}$ for 15 years old hybrid pine stand. Partial time models for each activity in the cycle were developed, and the influence of the thinning type, harvested species, number of products bucking, volume of trees, and feller forms were determined for each cycle activity. The adjusted models were integrated into a model of effective productivity achieving a $\mathrm{R}^{2} \mathrm{Aj}$ of 0.61 . The tree volume was significant to explain the time consumption of all evaluated activities, being especially crucial in felling, delimbing, and bucking the trees. The thinning type affected location time, while the species affected

1 Ingeniero Forestal, Becario Doctoral, Universidad Nacional de Misiones - CONICET - FCF, Calle Bertoni, 124, CEP 3380, Eldorado (MN), Argentina. eduardohildt@gmail.com

2 Ingeniero Forestal, Dr., Profesor Asociado de Explotación Forestal, Universidad Nacional de Misiones - FCF, Calle Bertoni, 124, CEP 3380, Eldorado (MN), Argentina. patricio.macdonagh@gmail.com

3 Ingeniero Forestal, Becario, Universidad Nacional de Misiones - FCF, Calle Bertoni, 124, CEP 3380, Eldorado (MN), Argentina. franciscosomma@hotmail.com

4 Ingeniero Forestal, Actitud Forestal, Estudio de Ingeniería y Representaciones, Calle Congreso, 241 - Local 1, CEP 3380, Eldorado (MN), Argentina. actitudforestal@gmail.com

5 Ingeniero Forestal, Selva SRL, Calle Jujuy, 1732, CEP 3380, Eldorado (MN), Argentina. danielduran@selvasrl. net
\end{abstract}

Recebido para publicação em 14/03/2016 e aceito em 16/08/2017

Ci. Fl., v. 28, n. 4, out. - dez., 2018 
delimbing and bucking time. When producing up to three assorted products, it was required more time for delimbing and bucking. There was an increase in the number of products when third thinning was performed on hybrid pine with respect to Pinus taeda.

Keywords: Wheeled harvester; Pinus taeda; Hybrid pine.

\section{INTRODUCCIÓN}

Las operaciones de raleos en plantaciones de pino presentan el desafío de obtener la mayor rentabilidad, por lo que la cosecha debe ser desarrollada con la mayor eficiencia posible y al menor costo. En el nordeste argentino, los segundos y terceros raleos están poco planificados, y gran parte de las decisiones fundamentales quedan en manos del operador del harvester.

Las empresas contratistas de cosecha generalmente emplean un único modelo de harvester para diferentes situaciones de trabajo, desde primeros raleos con volumen medio individual (VMi) de $0,08 \mathrm{~m}^{3}$ a terceros raleos con VMi de $0,7 \mathrm{~m}^{3}$, similares a los encontrados en una corta final (MAC DONAGH et al., 2013b). Estos harvesters también suelen utilizarse tanto en raleos de Pinus taeda como de pino hibrido (Pinus elliottii var. elliottii x Pinus. caribaea var. hondurensis). Esta es una actividad de gran complejidad y baja rentabilidad, donde se deben cosechar los árboles a extraer minimizando los daños a la masa remanente. La heterogeneidad existente en las forestaciones intervenidas implica que el harvester elabore árboles con volúmenes individuales muy dispares, generando diferentes combinaciones de productos. Por esta razón resulta importante contar con modelos de productividad que describan el comportamiento de los equipos bajo un amplio rango de volúmenes y diferentes situaciones de trabajo.

Diferentes estudios han demostrado que el volumen de los árboles cosechados es el principal factor que afecta a la productividad de la cosecha con harvester (MARTINS; SEIXAS; STAPE, 2009; MAC DONAGH et al., 2013a). Visser y Spinelli (2012), indican que el incremento de la productividad tiende a ser decreciente a partir de un volumen determinado debido a las dificultades de elaboración que presentan los árboles de gran tamaño.

Por otra parte, el tiempo requerido por el harvester para desplazarse y alcanzar los árboles a cosechar depende principalmente del tipo de raleo realizado, el distanciamiento entre los árboles y la cantidad de líneas que se cosechan simultáneamente (OVASKAINEN; UUSITALO; VÄÄTÄINEN, 2004; MARTINS; SEIXAS; STAPE, 2009). Además, el tiempo que el harvester emplea en el desrame y trozado de los árboles está determinado por la forma y largo de los fustes, así como por la cantidad y características de las ramas de cada especie. Por otra parte, la elaboración de varios productos requiere movimientos adicionales de la máquina y la grúa para ubicar las trozas en las pilas correspondientes, incrementando el tiempo de elaboración y reduciendo la productividad (HILDT; MAC DONAGH, 2015).

En la actualidad, el acceso a los sistemas de medición y control incorporados en los harvesters, junto al desarrollo de cámaras filmadoras portátiles, han permitido aumentar el detalle y precisión de los estudios de tiempos y movimientos, reduciendo los costos y dificultades inherentes al trabajo de campo (STRANDGARD; WALSH; ACUNA, 2013). Esto posibilita el análisis detallado de las actividades productivas y permite el desarrollo de modelos de tiempos parciales con mayor sensibilidad hacia los factores que afectan a cada actividad del ciclo operacional (MCNEEL; RUTHERFORD, 1994; PUTTOCK; SPINELLI; HARTSOUGH, 2005; NURMINEN; KORPUNEN; UUSITALO, 2006; MARTINS; SEIXAS; STAPE, 2009; NIEMISTÖ et al., 2012).

El objetivo de este estudio fue analizar las diferencias de productividad para harvesters de ruedas en segundos y terceros raleos de plantaciones de Pinus taeda y de pino hibrido, en relación con las características de las plantaciones y los productos obtenidos. 


\section{MATERIALES Y MÉTODOS}

\section{Características del área de estudio}

Los estudios de tiempos y movimientos fueron desarrollados en operaciones de segundo y tercer raleo en forestaciones de Pinus taeda y pino hibrido de la zona norte de la provincia de Misiones, en plantaciones cercanas a la ciudad de Puerto Esperanza ( $26^{\circ} 2^{\prime}$ S, $\left.54^{\circ} 38^{\prime} \mathrm{O}\right)$. Estos sitios presentan terrenos suavemente ondulados con pendientes entre el 0 y $5 \%$ y sus suelos pertenecen al gran grupo de los Kandiudultes, conocidos como "tierras coloradas" (PEÑA ZUBIATE; PINEDO, 1990).

Se evaluaron cuatro situaciones de trabajo: Segundos raleos de Pinus taeda (2T) con 12 años de edad, 24,5 cm de diámetro medio (DAPm), 19,1 m de altura media (Hm) y 0,48 $\mathrm{m}^{3}$ de VMi; Segundos raleos de pino hibrido $(2 \mathrm{H})$ con 10 años, 23,1 cm de DAPm, 16,3 m de Hm, y 0,42 $\mathrm{m}^{3}$ de VMi; Terceros raleos de Pinus taeda (3T) con 13 años, $30 \mathrm{~cm}$ de DAPm, 20,9 $\mathrm{m}$ de Hm, y 0,77 $\mathrm{m}^{3}$ de VMi; y Terceros raleos de pino hibrido $(3 \mathrm{H})$ con 15 años, $31,8 \mathrm{~cm}$ de DAPm, 24,1 $\mathrm{m}$ de Hm, y 1,02 $\mathrm{m}^{3}$ de VMi.

En las situaciones 2T, 3T y 3H se evaluó un harvester Logman $801 \mathrm{H}$ (HV 1), con neumáticos y tracción $4 \times 4$, equipado con un cabezal cosechador KETO 150 Supreme y sistema de control Motomit IT 6,5 " Serie E. Esta máquina base cuenta con $125 \mathrm{~kW}$ de potencia nominal, un peso total de $12000 \mathrm{~kg}$ y un caudal de fluido hidráulico de $2101 . \mathrm{min}^{-1}$. La grúa de barras paralelas tiene un alcance máximo de 10,3 m. El cabezal procesador tiene un peso de $1.040 \mathrm{~kg}$, un diámetro máximo de desrame de $0,45 \mathrm{~m}$ y una velocidad de alimentación máxima de $3,8 \mathrm{~m} \cdot \mathrm{s}^{-1}$. El operario de este equipo cuenta con 5 años de experiencia en la realización de raleos mecanizados.

En la situación restante (segundo raleo de pino hibrido $2 \mathrm{H}$ ) se evaluó un equipo de similares características (HV 2), con una grúa telescópica con $9.3 \mathrm{~m}$ de alcance y equipado con un cabezal KETO 150, de $810 \mathrm{~kg}$ de peso y una velocidad de alimentación máxima de $4 \mathrm{~m} \cdot \mathrm{s}^{-1}$. Este equipo cuenta con un sistema de control EPEC 4W50. El HV 2 fue introducido en el estudio debido a que un desperfecto en el sistema de control del HV 1 impidió evaluarlo en la situación 2H. El operador de este equipo cuenta con 7 años de experiencia.

Para ambos equipos el sistema de control efectuó las mediciones de longitud y diámetro por medio de sensores ubicados en los rodillos y centro del cabezal, presentando la información al operador mediante una pantalla ubicada dentro de la cabina. A los efectos de este estudio se consideró que ambos equipos presentan un comportamiento similar durante la operación de cosecha.

Las situaciones evaluadas poseen intensidades de cosecha similares, respondiendo a una reducción del 50\% en el número de árboles, regla general utilizada en los raleos de la región. En la Tabla 1 se presenta un resumen de los valores de densidad, área basal y volumen existentes antes y después de las intervenciones.

TABLA 1: Intensidad de raleo en cada situación.

TABLE 1: Thinning intensity in each situation.

\begin{tabular}{|c|c|c|c|c|c|c|c|}
\hline \multirow[b]{2}{*}{ Sit. } & \multicolumn{2}{|l|}{$\begin{array}{l}\text { Densidad } \\
\left(\text { arb.ha }^{-1}\right)\end{array}$} & \multicolumn{2}{|l|}{$\begin{array}{c}\text { Área basal } \\
\left(\mathrm{m}^{2} \cdot \mathrm{ha}^{-1}\right)\end{array}$} & \multicolumn{2}{|l|}{$\begin{array}{l}\text { Volumen } \\
\left(\mathrm{m}^{3} \cdot \mathrm{ha}^{-1}\right)\end{array}$} & \multirow[b]{2}{*}{$\begin{array}{l}\text { 1 Volumen extraído } \\
\qquad(\%)\end{array}$} \\
\hline & Inicial & Final & $\begin{array}{l}\text { Antes del } \\
\text { raleo }\end{array}$ & $\begin{array}{l}\text { Después del } \\
\text { raleo }\end{array}$ & $\begin{array}{l}\text { Antes del } \\
\text { raleo }\end{array}$ & $\begin{array}{l}\text { Después del } \\
\text { raleo }\end{array}$ & \\
\hline $3 \mathrm{H}$ & 372 & 256 & 30,1 & 25,2 & 380,2 & 139,7 & $63,30 \%$ \\
\hline $3 \mathrm{~T}$ & 367 & ---- & 32,6 & 26,2 & 284,1 & 96,6 & $66,00 \%$ \\
\hline $2 \mathrm{H}$ & 831 & 351 & 35,9 & 18,3 & 263,3 & 116,3 & $55,80 \%$ \\
\hline $2 \mathrm{~T}$ & 640 & 385 & 39,7 & 25,1 & 300,2 & 97 & $67,70 \%$ \\
\hline
\end{tabular}


Debido a que la situación $2 \mathrm{H}$ no contó con vías de extracción abiertas en el primer raleo, se efectuó un raleo "mixto" donde el harvester ingresó cortando de forma sistemática todos los árboles de la quinta línea y elaborando selectivamente los árboles marcados en las dos líneas adyacentes a cada lado del equipo. En cambio, en las demás situaciones donde ya existían vías de extracción separadas por entre 9 y 11 líneas, se realizó un raleo puramente selectivo sobre las vías de extracción existentes y un raleo "mixto" abriendo nuevas vías de extracción donde fueron necesarias. Para cada ciclo de trabajo se identificó si el harvester se encontraba posicionado sobre una vía de extracción nueva o una existente (raleo mixto o selectivo).

En las operaciones de segundo raleo se elaboraron trozas para aserrío con diámetro en punta fina (Dpf) mayor a $16 \mathrm{~cm}$ en Pinus taeda y mayor a $12 \mathrm{~cm}$ en pino hibrido, y trozas destinadas a trituración con Dpf mayor a $5 \mathrm{~cm}$. En las operaciones de tercer raleo se elaboraron trozas aserrables de hasta 4,25 m de longitud con Dpf mayor a $16 \mathrm{~cm}$, trozas destinadas a debobinado con Dpf mayor a $25 \mathrm{~cm}$ y trozas destinadas a trituración con Dpf mayor a $5 \mathrm{~cm}$.

\section{Recolección de datos}

El estudio fue realizado bajo condiciones de trabajo normales, sin que el observador interfiriera en la operación, por medio de cámaras filmadoras digitales instaladas dentro de la cabina de las máquinas evaluadas. Se grabaron de forma simultánea y continua los movimientos del cabezal cosechador y la información suministrada por la pantalla del sistema de control. Los videos fueron analizados manualmente con un software desarrollado a tal efecto, aplicando la metodología de toma de tiempos continuos (PARRA; CAREY, 2000; NIEMISTÖ et al., 2012; STRANDGARD; WALSH; ACUNA, 2013; ALAM et al., 2014).

La unidad experimental considerada fue el ciclo operacional definido como las actividades necesarias para la elaboración completa de un árbol. Durante el análisis de las filmaciones se determinó el inicio de las actividades que componen el ciclo operacional: inicio de la búsqueda de un árbol, inicio del corte, inicio del procesamiento, corte de cada troza e inicio de un nuevo ciclo. En base al momento de ocurrencia de estos eventos se calculó la duración de cada actividad productiva: búsqueda del árbol y ubicación del cabezal, que comprende desde la liberación del árbol anterior hasta el accionamiento de la sierra para el corte y volteo; volteo, desde el accionamiento de la sierra y el volteo del árbol y hasta el inicio del desplazamiento del cabezal para realizar el procesamiento del fuste; desrame y trozado, desrame del fuste y corte de todas las trozas elaboradas; y liberación de las ramas: desde el corte de la última troza hasta la liberación final de la copa remanente. Las distintas actividades complementarias y no productivas (movimientos adicionales, paradas, carreteo sobre camino) fueron medidas y discriminadas del análisis.

El número mínimo de observaciones necesarias fue calculado mediante (1) para proporcionar un error relativo de muestreo máximo del 5\% (BARNES, 1977; LEVY; LEMESHOW, 2008).

$$
n \geq\left(\mathrm{t}^{2} * \mathrm{cv}^{2}\right) / \mathrm{e}^{2}
$$

En que: $\mathrm{t}=$ Valor de la distribución t Student, con un nivel de confianza del 95\%; cv = Coeficiente de variación porcentual; e = Error admisible en porcentaje (5\%); $\mathrm{n}=$ Tamaño mínimo de la muestra.

Los diámetros en punta fina y punta gruesa y los largos de las trozas elaboradas fueron obtenidos de la información presentada por el sistema de control del harvester. Para estimar el DAP y la altura de los árboles se ajustaron índices de conicidad y funciones hipsométricas para cada sitio en particular. El volumen con corteza de cada árbol (Vol. Est.) fue estimado mediante modelos desarrollados para la región por Crechi et al. (2006) para Pinus taeda y por Costas et al. (2006) para pino hibrido. El volumen individual de las trozas elaboradas fue calculado mediante la fórmula de Smalian (PRODAN et al., 1997).

\section{Desarrollo de modelos}

Se ajustaron modelos mixtos de regresión para estimar el tiempo insumido en cada una de las actividades que componen el ciclo operacional respecto de variables categóricas (especie, tipo de intervención, modalidad de raleo, aplicación de volteo anti-impacto), numéricas (volumen estimado de los árboles, número de productos elaborados) y las interacciones entre estas. Se incorporó el efecto aleatorio de la parcela de bosque intervenida con el objetivo de independizar los ciclos individuales de las repeticiones a nivel de lote. 
Las variables numéricas incorporadas fueron seleccionadas en base a estudios similares (NURMINEN; KORPUNEN; UUSITALO, 2006; MAC DONAGH et al., 2013a; STRANDGARD; WALSH; ACUNA, 2013), priorizándose aquellas de fácil determinación a campo y de manejo cotidiano por los profesionales encargados de la cosecha.

Los modelos desarrollados incorporan como principal variable predictora al volumen total individual estimado con las funciones locales debido a que esta es la principal herramienta de estimación disponible al momento de planificar la cosecha.

El ajuste y selección de los modelos mixtos se realizó según la metodología establecida por Zuur et al. (2009), utilizando el módulo de "Modelos lineales, generales y mixtos" disponible en Infostat combinado con R (INFOSTAT, 2015). Los modelos finales fueron seleccionados buscando minimizar los valores del Criterio de Información de Akaike (AIC), el Criterio Bayesiano de Información (BIC), el logaritmo de la verosimilitud (Log-likelihood) y aumentar el coeficiente de determinación ajustado $\left(\mathrm{R}^{2} \mathrm{Aj}\right)$. Además, se tuvo en cuenta que todas las variables e interacciones incorporadas fueran significativas y que se observara una distribución normal y homocedástica de los residuos. Para ello se aplicó una transformación logarítmica a las variables dependientes de los modelos.

Los modelos de tiempos parciales por actividad fueron luego combinados de forma aditiva para obtener un modelo general del tiempo total del ciclo. Realizando el cociente entre el volumen aprovechado de los árboles y los tiempos predichos por esta ecuación se desarrolló un modelo para la productividad horaria efectiva (NURMINEN; KORPUNEN; UUSITALO, 2006; NIEMISTÖ et al., 2012), determinada en metros cúbicos con corteza cosechados por hora de trabajo efectiva (2).

PEF $=$ Vol.Aprov. $/($ TAE /3600)

En que: PEF $=$ Productividad del harvester por hora de trabajo efectivo $\left(\mathrm{m}^{3} \cdot \mathrm{h}^{-1}\right)$; Vol. Aprov. $=$ Volumen total aprovechado $\left(\mathrm{m}^{3}\right) ; \mathrm{TAE}=$ Tiempo total efectivo de elaboración $(\mathrm{s})$.

\section{RESULTADOS Y DISCUSIÓN}

Las situaciones de tercer raleo tuvieron un volumen medio similar para los árboles aprovechados mientras que los segundos raleos presentaron diferencias significativas, siendo los árboles de Pinus taeda $20 \%$ mayores que los de pino hibrido. En la Tabla 2 puede observarse un resumen del número de ciclos evaluados y su duración media. La Tabla 3 presenta las principales características dasométricas de los árboles cosechados.

TABLA 2: Resumen de los ciclos evaluados en cada situación.

TABLE 2: Cycle summaries for each situation.

\begin{tabular}{cccccc}
\hline & & \multicolumn{2}{c|}{$\begin{array}{c}\text { Volumen } \\
\text { Aprovechado } \\
\left(\mathrm{m}^{3}\right)\end{array}$} & & \multicolumn{2}{c}{ Tiempo Total del ciclo (s) } \\
\cline { 3 - 6 } Sit. & Ciclos validos & Media & SD & Media & SD \\
\hline $3 \mathrm{H}$ & 335 & $0,75 \mathrm{a}$ & 0,22 & 66,73 & 18,61 \\
$3 \mathrm{~T}$ & 422 & $0,71 \mathrm{a}$ & 0,18 & 57,22 & 16,17 \\
$2 \mathrm{H}$ & 574 & $0,36 \mathrm{c}$ & 0,16 & 43,28 & 14,4 \\
$2 \mathrm{~T}$ & 523 & $0,43 \mathrm{~b}$ & 0,16 & 44,72 & 12,05 \\
\hline
\end{tabular}

En que: $\mathrm{SD}=$ Desvío estándar. Letras diferentes indican diferencias significativas a una probabilidad $\mathrm{P}<0,05$. 
TABLA 3: Valores dasométricos medios para los árboles aprovechados.

TABLE 3: Harvested trees characteristics.

\begin{tabular}{ccccc}
\hline Sit. & $\begin{array}{c}\text { DAP } \\
(\mathrm{cm})\end{array}$ & $\begin{array}{c}\text { Altura } \\
(\mathrm{m})\end{array}$ & $\begin{array}{c}\text { Largo Aprovechado } \\
(\mathrm{m})\end{array}$ & $\begin{array}{c}\text { Vol. Est. Total } \\
\left(\mathrm{m}^{3}\right)\end{array}$ \\
\hline $3 \mathrm{H}$ & $26,9 \mathrm{c}$ & $22,59 \mathrm{~d}$ & $19,75 \mathrm{c}$ & $0,676 \mathrm{c}$ \\
$3 \mathrm{~T}$ & $27,9 \mathrm{~d}$ & $21,77 \mathrm{c}$ & $16,61 \mathrm{~b}$ & $0,663 \mathrm{c}$ \\
$2 \mathrm{H}$ & $20,9 \mathrm{a}$ & $17,65 \mathrm{a}$ & $14,51 \mathrm{a}$ & $0,333 \mathrm{a}$ \\
$2 \mathrm{~T}$ & $22,8 \mathrm{~b}$ & $19,43 \mathrm{~b}$ & $14,39 \mathrm{a}$ & $0,402 \mathrm{~b}$ \\
\hline
\end{tabular}

En que: DAP = Diámetro a la altura del pecho; Vol. Est. Total $=$ Volumen estimado total. Letras diferentes indican diferencias significativas a una probabilidad $\mathrm{P}<0,05$.

\section{Movimiento y ubicación}

El movimiento de la máquina y la ubicación del árbol a cosechar comprende el tiempo que requiere la máquina para desplazarse hasta una nueva ubicación, el tiempo que requiere el operador para localizar un árbol que se encuentre marcado para la cosecha o decidir si un árbol no marcado debe ser cortado para mejorar la distribución de los árboles remanentes en el rodal, y por último el movimiento de la grúa para posicionar el cabezal cosechador en la base del árbol a cortar. Esta actividad representó en promedio un 34,3\% del tiempo total del ciclo, una proporción cercana a la que encontraron Nurminen, Korpunen y Uusitalo (2006) para el movimiento y ubicación en raleos de Pinus sylvestris en Finlandia. En la situación $2 \mathrm{H}$ esta actividad tuvo una duración 3,2 segundos (s) menor que en 2T y representó únicamente el 29\% del tiempo operacional (frente al 37,9\% para 2T). Esto puede explicarse debido a la participación de un operador diferente, ya que las diferencias encontradas resultan similares a las observadas por Ovaskainen, Uusitalo, Väätäinen (2004) que hallaron diferencias de hasta el 30\% entre operadores que realizaban la ubicación de árboles, debido a las diferentes maneras en que cada operario planifica los movimientos a realizar.

Los terceros raleos presentaron un tiempo de movimiento y ubicación $37,5 \%$ superior (significativamente diferentes con una probabilidad $\mathrm{p}<0,05)$ a los segundos raleos, debido al mayor distanciamiento de los árboles cosechados y la existencia de un sotobosque más denso.

En los terceros raleos no se observaron diferencias significativas en el tiempo de ubicación para los árboles que se encontraban al frente o inmediatamente al lado del harvester. En cambio, el alcance de los árboles de las segundas y terceras líneas requirió un tiempo 7,4\% mayor. En segundos raleos el alcance de los árboles de las líneas exteriores requirió un tiempo 20,9\% mayor que los de la línea central, mientras que los árboles adyacentes al harvester requirieron un tiempo 11,3\% menor que los centrales. Esto puede explicarse por la mayor densidad de árboles encontrada en los segundos raleos, restringiendo la movilidad de la grúa para evitar daños a los árboles de la línea adyacente al alcanzar aquellos que se encontraban más alejados.

En la realización de raleos puramente selectivos el tiempo medio de ubicación y movimiento se incrementó un 4\% respecto de las situaciones de raleo mixto, donde el harvester realizó el corte sistemático de una línea y el corte selectivo de los árboles marcados en las líneas adyacentes. Este incremento se debe a que en los sectores donde se realizaron raleos puramente selectivos no hubo árboles ubicados al frente de la máquina sobre la vía de extracción, por lo que la importancia relativa de los árboles de las filas exteriores fue mayor.

El modelo mixto de regresión elegido incorpora los efectos del tipo de intervención, la forma de realización del raleo y el volumen de los árboles (3). Todos los términos resultaron significativos a una probabilidad $\mathrm{p}<0,05$, consiguiendo explicar un $34 \%$ de la variabilidad observada $\left(\mathrm{R}^{2} \mathrm{Aj}=0,337\right)$.

$$
\ln (T U B)=2,3956+0,3186 *(3 e r \text { Raleo })+0,0412 *(\text { Raleo Selectivo })+0,5076 * \text { Vol. Est. }
$$

En que: $\ln (\mathrm{TUB})=$ Logaritmo natural del tiempo de ubicación; 3er Raleo = Variable Dummy que toma el valor 1 para los ciclos medidos en tercer raleo y 0 para segundo raleo; Raleo Selectivo = Variable 
Dummy que toma el valor 1 para los ciclos en los que se realizó raleo puramente selectivo y 0 para raleo mixto; Vol.Est. = Volumen individual estimado de los árboles.

Independientemente de las situaciones evaluadas se observó un aumento exponencial en el tiempo de ubicación al incrementarse el volumen de los árboles debido a las dificultades existentes al posicionar el cabezal cosechador alrededor del fuste en los árboles de mayor tamaño (Figura 1 A). Sin embargo, Nurminen, Korpunen y Uusitalo (2006), comparando raleos mecanizados y cortas finales de bosques mixtos de Pinus sylvestris y Picea abies, no encontraron diferencias significativas ni tampoco relación entre el tiempo de posicionamiento del cabezal y el tamaño de los árboles, el número de árboles cosechados o las características del sitio.

\section{Corte y volteo}

Las actividades de corte y volteo comprendieron el accionamiento de la sierra al iniciar el corte del árbol, la caída del árbol acompañada por el movimiento del cabezal hasta su posición horizontal y el inicio del movimiento de los rodillos para desplazar el cabezal sobre el fuste. Esta actividad constituyó en promedio un $14 \%$ del tiempo total de operación, sin presentar diferencias significativas entre especies o tipo de raleo. Esta proporción fue similar al 18\% encontrado por Nurminen, Korpunen y Uusitalo (2006) para la operación de corte.

El volteo en las líneas más alejadas requirió un tiempo significativamente mayor $(25,4 \%)$ que el correspondiente a la línea central y a las líneas adyacentes debido a la merma en la capacidad de carga de la grúa al operar cerca de su alcance máximo. Esto coincide con lo determinado por Mac Donagh et al. (2013a) en primeros y segundos raleos realizados en la región, al igual que por Ovaskainen, Uusitalo, Väätäinen (2004) en raleos de Pinus sylvestris, donde para los árboles situados a más de $5 \mathrm{~m}$ de las vías de extracción el volteo tardó 2 segundos más que para los árboles cercanos al harvester.

Para las operaciones de tercer raleo se observó que en los árboles con un VMi de $0,82 \mathrm{~m}^{3}$ (Desvío Estándar (SD): $0,18 \mathrm{~m}^{3}$ ) para pino hibrido y de $0,96 \mathrm{~m}^{3}$ (SD: 0,23 $\mathrm{m}^{3}$ ) para Pinus taeda el operario del harvester habitualmente realizó un apeo no controlado, liberando el árbol durante la caída para evitar la transmisión a la grúa del impacto generado por el golpe del árbol contra el suelo. Esta modalidad de volteo "anti-impacto" se observó en el 23,3\% de los ciclos evaluados en la situación $3 \mathrm{H}$ y en el 6,9\% de los ciclos para 3T. Esta diferencia se debió a que los árboles de pino hibrido poseen un fuste más largo, con un menor ángulo en la inserción de las ramas y una copa menos densa que los ejemplares de Pinus taeda, siendo menor el frenado de la caída por el enganche de la copa contra otros árboles y la rotura de las ramas al alcanzar el suelo.

Se observaron diferencias significativas en el tiempo de corte y volteo debido a la realización del volteo "anti-impacto", siendo este en promedio $130 \%$ mayor (16,9 s para $3 \mathrm{H}$ y $20,7 \mathrm{~s}$ para $3 \mathrm{~T}$ ) al requerido durante los ciclos de volteo normal $(7,6 \mathrm{~s}$ para $3 \mathrm{H}$ y $6,6 \mathrm{~s}$ para $3 \mathrm{~T})$ debido a los movimientos de la grúa necesarios para localizar y capturar nuevamente el fuste liberado durante la caída. En la Figura 2 (B y D) se observa como la aplicación del volteo anti-impacto en terceros raleos provoca un aumento en el tiempo total del ciclo y una reducción en la productividad efectiva de la operación. Aunque esta modalidad de trabajo permite sortear las dificultades que presenta este equipo al cosechar árboles de gran volumen, también demuestra que resulta inadecuado para la realización de terceros raleos donde el volumen medio de los árboles cosechados se aproxime a $1 \mathrm{~m}^{3}$.

El modelo mixto de regresión ajustado conservó como variables predictoras significativas el tipo de volteo aplicado y el volumen del árbol cosechado, alcanzando a explicar el $40 \%$ de la variabilidad observada $\left(R^{2} A j=0,399\right)$ (4).

$$
\ln (T V)=1,4731+0,8377 *(\text { Volt. anti }- \text { impacto })+0,6039 * \text { Vol. Est. }
$$

En que: $\ln (\mathrm{TV})=$ Logaritmo natural del tiempo de volteo; Volt. anti - impacto = Variable Dummy que toma el valor 1 cuando se realiza el volteo "anti-impacto" y el valor 0 cuando el volteo es normal. Vol. Est. $=$ Volumen individual estimado de los árboles. 
Como puede observarse en la Figura 1 B, el tiempo de volteo, aumenta proporcionalmente al incrementarse el volumen de los árboles para ambos tipos de volteo debido al mayor tiempo insumido en el corte y movimiento del fuste en árboles de mayor tamaño. Esta relación es similar a la obtenida por Nurminen, Korpunen y Uusitalo (2006) al ajustar un modelo lineal en base al volumen de los árboles, consiguiendo explicar el $26 \%$ de la variabilidad observada en cosechas finales con volúmenes comprendidos entre 0,2 y $1,2 \mathrm{~m}^{3}$
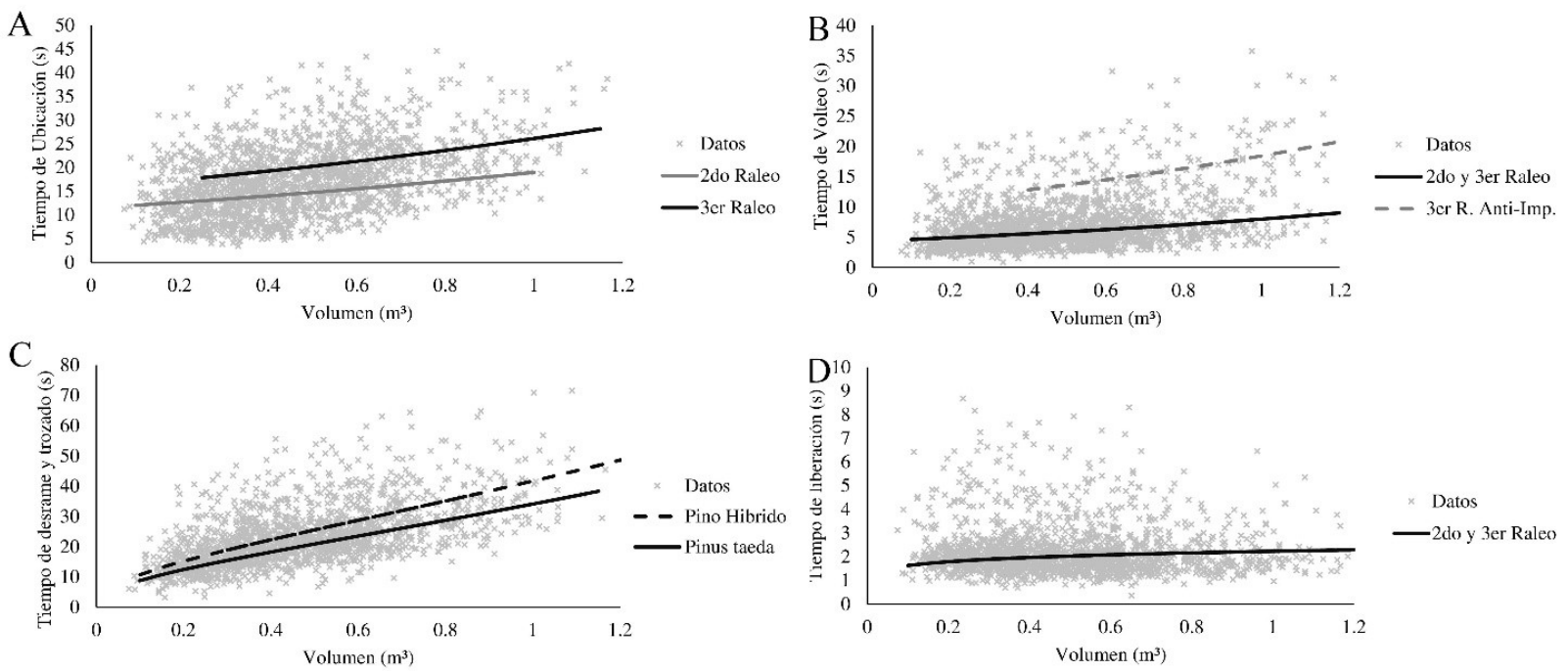

FIGURA 1: Comportamiento de los modelos predictivos parciales desarrollados para las distintas situaciones evaluadas.

FIGURE 1: Partial predictive model behavior in thinning operations.

\section{Desrame y trozado}

Esta operación comprende el tiempo transcurrido desde el accionamiento de los rodillos alimentadores para desplazar el cabezal sobre el fuste, el desrame del árbol y el trozado de los diferentes productos elaborados, finalizando con el corte de la última troza comercial, representando en promedio el $47 \%$ del tiempo del ciclo de elaboración.

El tiempo de desrame y trozado de los árboles varió significativamente entre las especies evaluadas, siendo un $22 \%$ mayor en el caso del pino hibrido, debido a que se obtuvo una cantidad $13 \%$ mayor de trozas en comparación con Pinus taeda, para un mismo VMi y cantidad de productos trozados, lo que implicó un mayor tiempo de accionamiento de la sierra y movimiento de la grúa.

Por otra parte, en terceros raleos se observaron diferencias significativas en la longitud total del fuste aprovechado para Pinus taeda y pino hibrido independientemente del diámetro de los árboles. Estos últimos presentaron una longitud de fuste aprovechado $15 \%$ mayor que los de Pinus taeda, por lo que se obtuvieron un mayor número de trozas por fuste, implicando un mayor tiempo de elaboración.

El número de productos elaborados por el equipo estuvo directamente relacionado con el tiempo de desrame y trozado de los fustes, siendo este un $10 \%$ superior al elaborarse dos productos (trozas para aserrío y trozas para trituración) frente a la elaboración de un único producto (trozas para trituración). Del mismo modo, la elaboración de 3 productos (dos clases para aserrío y una para trituración) requirió un tiempo 21\% mayor que un único producto. Este aumento del tiempo requerido por la actividad se debió al movimiento de la grúa necesario para clasificar los productos elaborados en diferentes pilas.

El tiempo de desrame y trozado aumentó de forma directa con el volumen de los árboles debido a que los árboles más grandes tuvieron un fuste más largo y produjeron un mayor número de trozas cortadas y clasificadas por el cabezal cosechador. Además, el aumento en el peso de los fustes requirió mayores esfuerzos de la grúa y el cabezal procesador. 
El modelo de regresión lineal múltiple ajustado incorporó los efectos de la especie, el número de productos elaborados y el volumen de los árboles, explicando el $51 \%$ de la variabilidad observada en el tiempo de desrame y trozado $\left(\mathrm{R}^{2} \mathrm{Aj}=0,505\right)(5)$.

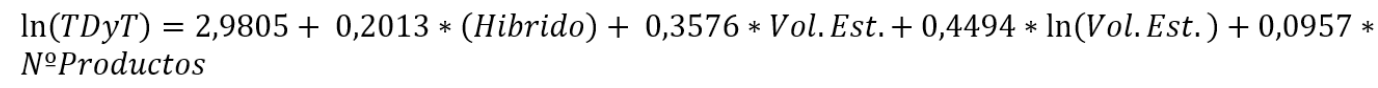

En que: $\ln ($ TDyT $)=$ Logaritmo natural del tiempo de desrame y trozado; Hibrido $=$ Variable Dummy que toma el valor 1 cuando se cosechó Pino Hibrido y el valor 0 cuando se cosechó Pinus taeda. Vol.Est. $=$ Volumen individual estimado de los árboles, $\mathrm{N}^{\mathrm{o}}$ Productos $=$ Cantidad de productos diferentes elaborados para ese árbol.

Como referencia, Nurminen, Korpunen y Uusitalo (2006) desarrollaron un modelo parcial que consiguió explicar el $54 \%$ de la variabilidad del tiempo de procesamiento para cortas finales de Pinus sylvestris, obteniendo una función de tendencia exponencial debido a las limitaciones observadas en la maquina al procesar árboles grandes.

\section{Liberación de la copa}

Esta actividad comprendió le tiempo transcurrido entre el corte de la última troza elaborada hasta la apertura del cabezal cosechador y la caída de la punta del fuste no aprovechable. El tiempo de liberación representó aproximadamente el 4,5\% del tiempo total del ciclo operacional.

El modelo elegido incorporó como variables predictoras significativas al volumen del árbol y el número de productos elaborados, consiguiendo explicar el $6 \%$ de la variabilidad total $\left(R^{2} A j=0,058\right)(6)$. El bajo nivel de explicación alcanzado se debe a la corta duración de esta actividad $(<2,5 \mathrm{~s})$, determinada principalmente por las decisiones del operador, presentando una baja correlación con el volumen de los árboles.

$$
\ln (T L)=1,006+0,1376 * \ln (\text { Vol.Est. })-0,1014 * \text { NoProductos }
$$

En que: $\ln (\mathrm{TL})=$ Logaritmo natural del tiempo de liberación; Vol.Est. $=$ Volumen individual estimado de los árboles, $\mathrm{N}^{\mathrm{o}}$ Productos= Cantidad de productos diferentes elaborados para ese árbol.

La relación positiva observada entre el tiempo de liberación y el volumen de los árboles se debe a que los árboles de mayor volumen generalmente presentan una copa más voluminosa y una mayor acumulación de ramas desplazadas por el cabezal durante el desrame del fuste.

La elaboración de un mayor número de productos requirió un tiempo de liberación significativamente menor. Esto puede deberse a que cuando se elaboraron diferentes productos aserrables las trozas destinadas a trituración fueron obtenidas principalmente de la punta fina del fuste, reduciendo la cantidad de material remanente que debe ser liberado por el cabezal.

\section{Tiempo total de elaboración}

Se observaron diferencias significativas entre las situaciones evaluadas para el tiempo total del ciclo de elaboración. $3 \mathrm{H}$ presentó un tiempo medio total por ciclo de 66,7 segundos (SD: 18,6 s) para un volumen aprovechado medio de $0,75 \mathrm{~m}^{3}$. La situación $3 \mathrm{~T}$, con un volumen medio aprovechado similar $(0,71$ $\mathrm{m}^{3}$ ) se diferenció significativamente de la anterior con un tiempo total de 57,2 s (SD: 16,2 s). No se observaron diferencias significativas entre las situaciones de segundo raleo (Tabla 4). 
TABLA 4: Valores medios de los tiempos por actividad para las distintas situaciones evaluadas. TABLE 4: Average activity times in each thinning operation.

\begin{tabular}{cccccccc}
\hline & $\begin{array}{c}\text { Volumen } \\
\text { Aprovechado }\end{array}$ & Ubicación & Volteo & $\begin{array}{c}\text { Desrame } \mathrm{y} \\
\text { trozado }\end{array}$ & Liberación & Total & Prod. Ef. \\
Sit. & $\left(\mathrm{m}^{3}\right)$ & $(\mathrm{s})$ & $(\mathrm{s})$ & $(\mathrm{s})$ & $(\mathrm{s})$ & $(\mathrm{s})$ & $\left(\mathrm{m}^{3} \cdot \mathrm{hef}^{-1}\right)$ \\
\hline $3 \mathrm{H}$ & 0,75 & $23,7 \mathrm{~d}$ & $9,8 \mathrm{c}$ & $31,1 \mathrm{~d}$ & $2,1 \mathrm{a}$ & $66,7 \mathrm{c}$ & $40,5 \mathrm{c}$ \\
$3 \mathrm{~T}$ & 0,71 & $20,8 \mathrm{c}$ & $7,6 \mathrm{~b}$ & $26,8 \mathrm{c}$ & $2,1 \mathrm{a}$ & $57,2 \mathrm{~b}$ & $44,7 \mathrm{~d}$ \\
$2 \mathrm{H}$ & 0,36 & $12,5 \mathrm{a}$ & $6,1 \mathrm{a}$ & $22,1 \mathrm{~b}$ & $2,5 \mathrm{~b}$ & $43,3 \mathrm{a}$ & $29,9 \mathrm{a}$ \\
$2 \mathrm{~T}$ & 0,43 & $16,9 \mathrm{~b}$ & $6,2 \mathrm{a}$ & $19,5 \mathrm{a}$ & $2,1 \mathrm{a}$ & $44,7 \mathrm{a}$ & $34,6 \mathrm{~b}$ \\
\hline
\end{tabular}

Letras diferentes indican diferencias significativas a una probabilidad $\mathrm{P}<0,05$ ajustadas considerando al volumen aprovechado como covariable.

Los valores predichos para el tiempo total se obtuvieron sumando los modelos individuales ajustados para cada actividad, previa aplicación del antilogaritmo a la variable respuesta (7). La combinación de los diferentes modelos parciales permite explicar el $61 \%$ de la variabilidad observada en el tiempo total de elaboración para ambas especies e intervenciones $\left(\mathrm{R}^{2}\right.$ Ajustado para los 15 parámetros que componen el modelo $=0,609$ ). Este nivel de explicación alcanzado resulta superior al obtenido individualmente para las distintas actividades parciales. La construcción de este modelo resulta similar a la desarrollada por Nurminen, Korpunen y Uusitalo (2006), los cuales combinaron modelos de tiempos parciales por actividad con valores de tiempos medios para las actividades que no presentaron correlación significativa con el volumen.

Tiempo Total $(s)=e^{\ln (T U B)}+e^{\ln (T V)}+e^{\ln (T D y T)}+e^{\ln (T L)}$

En que: TUB = Tiempo de ubicación; TV = Tiempo de corte y volteo; TDyT = Tiempo de desrame y trozado; $\mathrm{TL}=$ Tiempo de liberación; e $=$ Base de los logaritmos naturales.

El comportamiento del modelo puede observarse en la Figura 2 A para las situaciones de segundo y tercer raleo considerando únicamente volteos normales. Las curvas presentadas muestran el tiempo predicho para la realización de raleos selectivos elaborando dos productos en árboles de distintos volúmenes.

\section{Productividad efectiva}

La mayor productividad se alcanzó en raleos de Pinus taeda con $34,6 \mathrm{~m}^{3} \cdot \mathrm{hef}^{-1}$ para $2 \mathrm{~T}$ y 44,7 $\mathrm{m}^{3}$.hef ${ }^{-1}$ para 3T (Tabla 4). Los raleos de pino hibrido presentaron productividades significativamente menores (18\% menor en tercer raleo y $5 \%$ menor en segundo raleo) debido principalmente al mayor tiempo insumido por la maquina en el desrame y trozado y a la existencia de un mayor número de ciclos con volteo anti-impacto.

Las productividades alcanzadas resultan superiores a las observadas para diámetros similares por McNeel y Rutherford (1994) en la cosecha selectiva de bosques naturales de Pinus ponderosa; por Niemisto et al. (2012) en la cosecha de Picea abies; y por Nurminen, Korpunen y Uusitalo (2006) para Pinus sylvestris.

La productividad por hora de trabajo efectiva fue estimada en base al volumen aprovechado por el harvester para cada árbol y a los tiempos totales obtenidos mediante los modelos parciales combinados. El modelo de tiempos totales permite explicar el $61 \%$ de la productividad efectiva observada $\left(\mathrm{R}^{2}\right.$ Ajustado para los 16 parámetros que componen el modelo $=0,609)(8)$. Este nivel de explicación resulta superior al $41 \%$ obtenido por Mac Donagh et al. (2013a) en segundos raleos de Pinus taeda.

$$
\text { Prod.Ef. }\left(\frac{m^{3}}{h}\right)=\frac{\text { Vol.Aprovechado }\left(m^{3}\right) * 3600\left(\frac{s}{h}\right)}{\text { Tiempo total }(s)}
$$

En que: Prod.Ef. $=$ Productividad efectiva; Vol.Aprovechado $=$ Volumen de las trozas obtenidas. Como puede observarse en la Figura 2 (A y C), mientras el tiempo de elaboración aumentó al 
incrementarse el tamaño de los árboles, la productividad efectiva del equipo redujo su crecimiento hasta alcanzar un máximo con árboles de aproximadamente $1 \mathrm{~m}^{3}$. Este máximo, siguiendo el razonamiento de Visser y Spinelli (2012), demuestra que el equipo no presenta limitaciones técnicas graves al cosechar árboles de segundos raleos, pero en cambio puede verse limitado al elaborar los árboles más grandes de los terceros raleos.

A

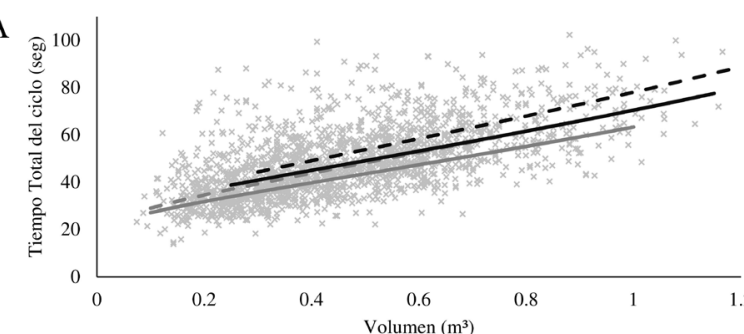

$\mathrm{C}$

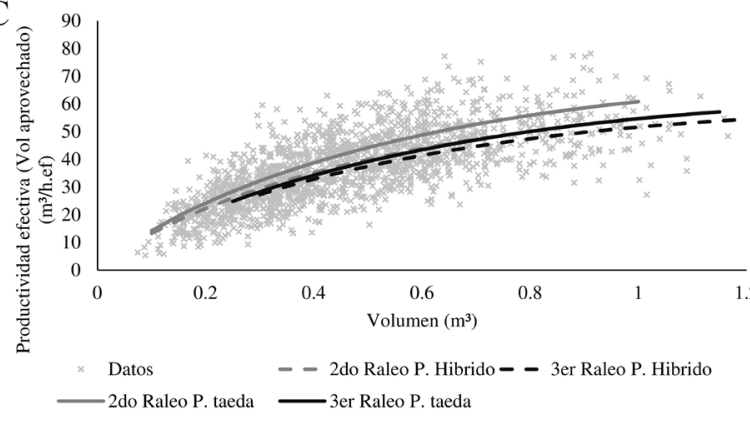

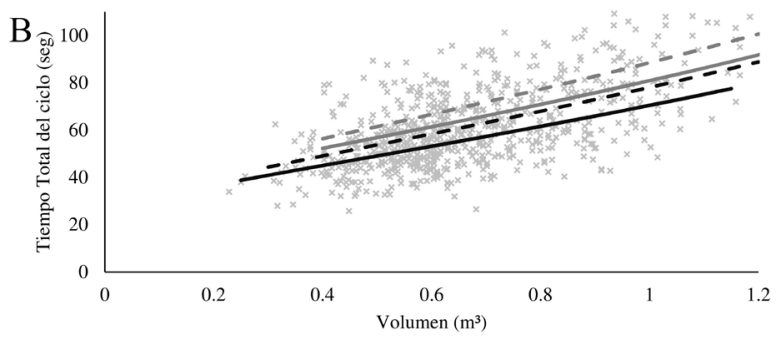

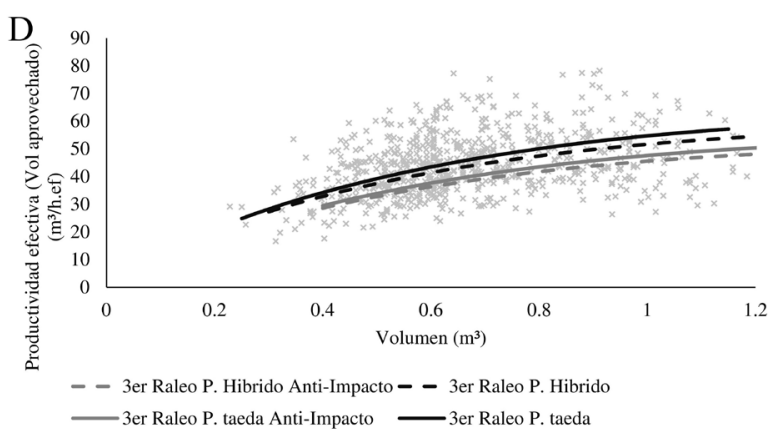

FIGURA 2: Comportamiento de los modelos predictivos del tiempo de operación total y la productividad efectiva. FIGURE 2: Time and productivity model behavior.

Por otra parte, tanto la elaboración de varios productos, como el incremento en el volumen de los árboles y la obtención de un mayor número de trozas por fuste para Pino hibrido representan la cosecha de mayor cantidad de madera rolliza de alto valor. En 2T, los árboles en los que se elaboró un único producto (Volumen medio: $0,35 \mathrm{~m}^{3}$ ) generaron $68 \%$ de material triturable y $32 \%$ de trozas aserrables de $3,65 \mathrm{~m}$ de longitud, con alto valor industrial. En cambio, cuando se elaboraron dos productos (Vol. Medio: 0,46 $\mathrm{m}^{3}$ ) se produjo $27 \%$ de madera destinada a trituración y $73 \%$ de trozas aserrables. Por otra parte, la elaboración de tres productos en $2 \mathrm{H}$ (Vol. medio: $0,48 \mathrm{~m}^{3}$ ) generó $20 \%$ de material triturable, $23 \%$ de trozas aserrables de $3,04 \mathrm{~m}$ de un valor intermedio y $56 \%$ de trozas aserrables de $3.95 \mathrm{~m}$ con alto valor industrial.

En 3H también se generó menor proporción de material triturable (15\%) que en 3T $(17 \%)$ para la elaboración de dos productos en árboles con similar VMi $\left(0,69 \mathrm{~m}^{3}\right.$ para $3 \mathrm{H}$ y $0,65 \mathrm{~m}^{3}$ para $\left.3 \mathrm{~T}\right)$. Para ambas especies la elaboración de 3 productos (Vol. Medio: $1 \mathrm{~m}^{3}$ ) significó una reducción de $6 \%$ en la proporción de material triturable de bajo valor obtenido, respecto a la elaboración de 2 productos (Vol. Medio: 0,67 m³).

\section{CONCLUSIONES}

El análisis individual de las distintas actividades que conforman el ciclo operacional permitió identificar los factores que afectan a cada actividad, posibilitando la construcción de un modelo de tiempos y productividad efectiva sensible al efecto de los factores de interés.

El tiempo de búsqueda y ubicación aumentó el incrementarse la distancia entre los árboles cosechados, el número de líneas cosechadas y el tamaño de los árboles que debe capturar el cabezal.

La operación de volteo dependió principalmente del volumen de los árboles aprovechados, observándose inconvenientes técnicos al realizar el volteo de árboles mayores a $0,8 \mathrm{~m}^{3}$ en terceros raleos, lo que determinó la aplicación de técnicas de volteo que protejan al harvester de impactos excesivos.

La operación de desrame y trozado fue la principal actividad del ciclo operacional y estuvo de- 
terminada por el volumen de los árboles. La elaboración de un mayor número de productos implico un aumento de hasta el $21 \%$ en el tiempo de trozado. Las características de forma del pino hibrido favorecieron una mayor longitud de fuste aprovechado, aumentando el número de trozas obtenidas y su tiempo de elaboración. Por otra parte, la elaboración de varios productos permitió obtener una mayor proporción de trozas con alto valor industrial, lo que compensaría la reducción en la productividad del harvester.

\section{AGRADECIMIENTOS}

Este trabajo fue parcialmente financiado por el ANR 155/12 del FONTAR y por una beca doctoral del CONICET. Agradecemos a la empresa SELVA SRL por facilitar el acceso a las situaciones evaluadas.

\section{REFERENCIAS}

ALAM, M. et al. A log-by-log productivity analysis of two Valmet 475EX harvesters. International Journal of Forest Engineering, [s. 1.], v. 25, n. 1, p. 14-22, mar. 2014.

BARNES, R. M. Estudo de movimentos e de tempos: projeto e medida do trabalho. São Paulo: Edgard Blucher, 1977. 635 p.

COSTAS, R. A. et al. Funciones de volúmenes del híbrido Pinus elliottii var. elliottii x Pinus caribaea var. hondurensis. In: JORNADAS TÉCNICAS FORESTALES Y AMBIENTALES, 12., 2006, Eldorado. Anais... Eldorado: INTA/FCF, 2006. p. 10.

CRECHI, E. et al. Modelos de estimación del volumen individual de árboles con y sin corteza de Pinus taeda L. para la zona de Norte de Misiones, Argentina. In: JORNADAS TÉCNICAS FORESTALES Y AMBIENTALES, 12., 2006, Eldorado. Anais... Eldorado: INTA/FCF, 2006. p. 14.

HILDT, E.; MAC DONAGH, P. M. Modelos de productividad para procesadores forestales en el noreste Argentino. Scientia Forestalis, Piracicaba, v. 43, n. 107, p. 561-571, set. 2015.

INFOSTAT. Versión 2015. Córdoba: Grupo InfoStat; FCA; Universidad Nacional de Córdoba, 2015.

LEVY, P. S; LEMESHOW, S. Sampling of populations: methods and applications. 4th ed. New York: John Wiley \& Sons, 2008. 578 p.

MAC DONAGH, P. M. et al. Influencia de la intensidad de raleos en la performance de un harvester de ruedas en el noreste Argentino. Floresta, Curitiba, v. 43, n. 4, p. 653-662, oct. 2013a.

MAC DONAGH, P. M. et al. Productividad y costos de pequeños "harvester" con oruga, operando en plantaciones de Pinus spp. en el noreste argentino. Scientia Forestalis, Piracicaba, v. 42, n. 99, p. 425-434. sep. 2013b.

MARTINS, R. J.; SEIXAS, F.; STAPE, J. L. Avaliação técnica e econômica de um harvester trabalhando em diferentes condições de espaçamento e arranjo de plantio em povoamento de eucalipto. Scientia Forestalis, Piracicaba, v. 37, n. 83, p. 253-263, sep. 2009.

MCNEEL, J. F.; RUTHERFORD, D. Modeling harvester-forwarder system performance in selection harvest. Journal of Forest Engineering, [s. 1.], v. 6, n. 1, p. 7-14, 1994.

NIEMISTÖ, P. et al. Impact and productivity of harvesting while retaining young understorey spruces in final cutting of downy birch. Silva Fennica, Joensuu, v. 46, n. 1, p. 81-97, 2012.

NURMINEN, T.; KORPUNEN, H.; UUSITALO, J. Time Consumption analysis of the mechanized cut-tolength harvesting system. Silva Fennica, Joensuu, v. 40, n. 2, p. 335-363, 2006.

OVASKAINEN, H.; UUSITALO, J.; VÄÄTÄINEN, K. Characteristics and significance of a Harvester Operator's Working technique in thinnings. International Journal of Forest Engineering, [s. 1.], v. 15, n. 2, p. 67-77, mar. 2004.

PARRA, M. O.; CAREY, P. B. Consideraciones metodológicas para la evaluación de la cortadora - procesadora (harvester) en operaciones forestales. Bosque, Valdivia, v. 21, n. 2, p. 137-146, 2000.

PEÑA ZUBIATE, C. A.; PINEDO, D. M. Atlas de suelos de la República Argentina. Buenos Aires: Secretaría de Agricultura, Ganadería y Pesca; Instituto Nacional de Tecnología Agropecuaria, 1990. v. $2.600 \mathrm{p}$.

PRODAN, M. et al. Mensura Forestal. San José: Instituto Internacional de Cooperación para la 
Agricultura; Gesellschaft für Technische Zusammenarbeit, 1997. 586 p.

PUTTOCK, D.; SPINELLI, R.; HARTSOUGH, B. R. Operational trials of cut-to-length harvesting of poplar in a mixed wood stand. International Journal of Forest Engineering, [s. 1.], v. 16, n. 2, p. 39-49, 2005. STRANDGARD, M.; WALSH, D.; ACUNA, M. Estimating harvester productivity in Pinus radiate plantations using StanForD stem files. Scandinavian Journal of Forest Research, Sweden, v. 28, n. 1, p. 73-80, jul. 2013.

VISSER, R.; SPINELLI, R. Determining the shape of the productivity function for mechanized felling and felling-processing. Journal of Forest Research, Tokio, v. 17, n. 5, p. 397-402, 2012.

ZUUR, A. F. et al. Mixed effects models and extensions in ecology with R. New York: Springer, 2009. 574 p. 